\title{
An optical time domain reflectometry set-up for laboratory work at École Supérieure d'Optique
}

G. Lucas-Leclin, T. Avignon, L. Jacubowiez

G. Lucas-Leclin, T. Avignon, L. Jacubowiez, "An optical time domain reflectometry set-up for laboratory work at Ecole Supérieure d'Optique," Proc. SPIE 9664, Ninth International Topical Meeting on Education and Training in Optics and Photonics, 96640X (24 October 2005); doi: 10.1117/12.2207695

SPIE Event: Ninth International Topical Meeting on Education and Training in Optics and Photonics, 2005, Marseille, France 


\title{
Ref ETOP037
}

\section{An Optical Time Domain Reflectometry Set-Up for Laboratory Work at École Supérieure d'Optique}

\author{
G. Lucas-Leclin, T. Avignon, L. Jacubowiez
}

Ecole Supérieure d'Optique, Centre Universitaire, Bat. 503, 91403 Orsay cedex, France e-mail : gaelle.lucas-leclin@iota.u-psud.fr

\section{Abstract}

We describe the optical time domain reflectometer for the characterization of single-mode fibers at $1.55 \mu \mathrm{m}$ that have been realized at École Supérieure d'Optique during students traineeships. Our set-up is an excellent educational tool to familiarize undergraduate students with optical telecommunication technologies as well as detection problems.

\section{Keywords :}

Optical Time Domain Reflectometry; Lab work at the University level.

\section{Introduction}

\section{Summary}

The Ecole Supérieure d'Optique (Orsay, France) offers top-level educational opportunities in engineering 5 years beyond secondary school education. The school is mainly concentrated on professions where optics plays an important role, and devotes considerable resources to both experimental work and teaching. We describe in the following an optical time domain reflectometer that have been realized by students under the direction of teachers in the laboratory, for training purpose. Optical Time Domain Reflectometry (OTDR) $)^{1,2}$ is a very common method to control optical fibers in transmission lines. It allows to test the whole length of a fiber from a single end, to locate precisely its defaults and measure its losses. Commercial OTDR instruments give these informations in a very simple way, but are not of pedagogical use since they do not give access to all the parameters of the set-up.

\section{Optical Time Domain Reflectometry}

The principle of OTDR measurements is very simple: short and intense light pulses from a laser diode are launched into the fiber under test via a directionnal coupler; pulses are reflected back on the defects inside the fiber, then detected through the coupler by a sensitive and fast photodiode. The time between the pulse emission and its detection gives the position of the defect inside the fiber under test, whereas the intensity of the reflection informs on its nature. Moreover, the measurement of the Rayleigh backscattering light gives information on the attenuation coefficient of the fiber.

Indeed, the backscattered signal power received from the launch point $z$ at which the scattering has occurred is given by (Eq. 1$)$ : 


$$
P_{b s}(z)=S \frac{\alpha_{d}}{2} v_{g} \tau \cdot P_{i n}(z=0) \cdot e^{-2 \alpha z}
$$

where $S$ is the capture coefficient, evaluated to $1.5 \times 10^{-3}$ in single-mode fibers, $\square_{d}$ is the diffusion coefficient of the fiber, $\square$ is its attenuation coefficient, $\square$ is the pulse duration and $P_{\text {in }}(z=0)$ is the signal power launched into the fiber. Of course the distance $z$ is directly related to the two-way time-of-flight $t$ of the light inside the fiber, expressed as $t=2 z / v_{g}$ with $v_{g}$ the group velocity of light inside the fiber. Therefore the temporal variations of the detected signal may be used to measure the attenuation coefficient $\square$ of the fiber under test.

It is obvious from the upon formula that the backscattered signal is proportional to the pulse duration $\square$. However high values for $\square$ reduce the resolution on the distance of defects; for example a $20 \mathrm{~m}$ resolution is achieved with a pulse duration of $100 \mathrm{~ns}$. Then the backscattered power from the output end of a typical $10 \mathrm{~km}$-long silica fiber is seven orders of magnitude lower than the launched optical power. Consequently the choice of the pulse duration is a compromise between signal sensitivity and resolution.

\section{Experimental set-up}

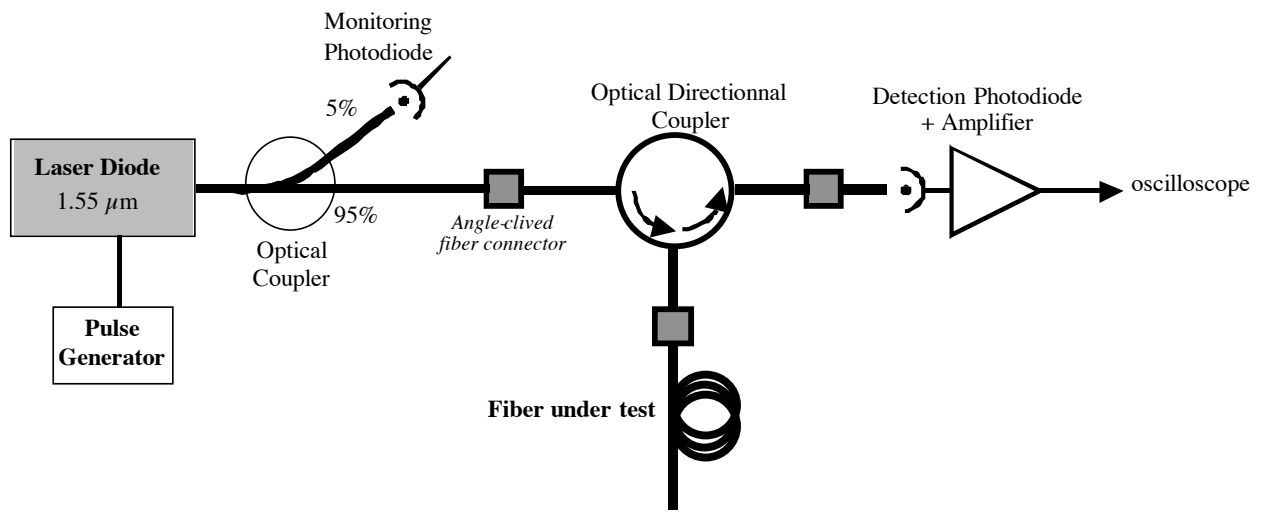

\section{Fig. 1 : Experimental set-up for Optical Time Domain Reflectometry}

In our set-up, the pulsed laser source is a fiber-coupled $1.55 \mu \mathrm{m}$ DFB laser with a maximal optical power of $20 \mathrm{~mW}$. The laser emission is controlled by an external pulse generator, and the pulse duration is variable between $10 \mathrm{~ns}$ and $10 \mu \mathrm{s}$. The photodetector module is based on a high-sped InGaAs PIN photodiode with a rise time of 0.5 ns. The photodetected signal is amplified in an home-made amplifier that provides enough gain (100 kV/W) to detect the low backscattering light as well as a fast response to detect the localized defects. Finally, the temporal variation of the backreflected light between two laser pulses is measured on a $400 \mathrm{MHz}$ Lecroy oscilloscope, and the measurements are analyzed on a computer. All optical and electrical signals are available for monitoring, and the duration as well as the repetition rate of the pulses are variable. 


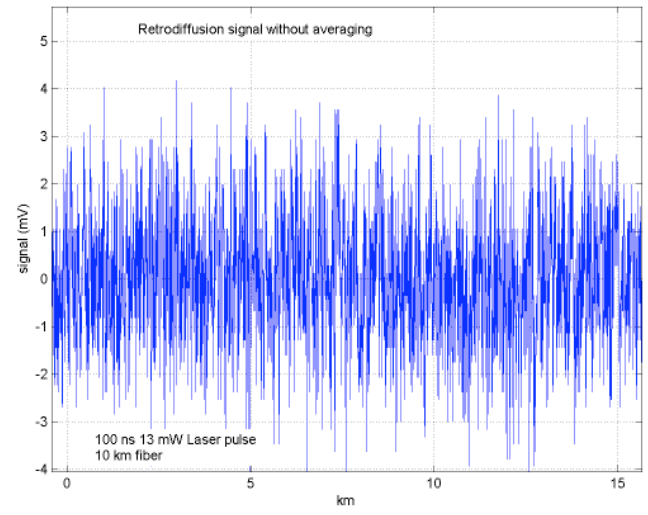

(a)

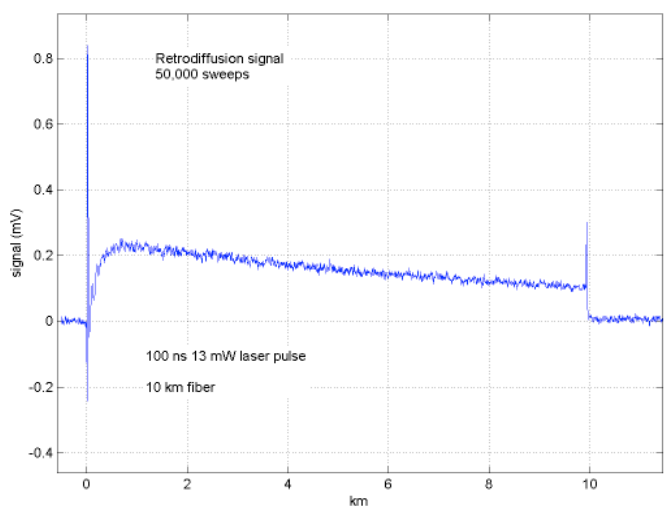

(b)

Figure 2 : Averaged backscattered signal from a $10 \mathrm{~km}$-long single-mode fiber; the pulse duraction is $100 \mathrm{~ns}$, and the laser peak power is $13 \mathrm{~mW}$. (a) detection signal; (b) same signal averaged over 50,000 sweeps.

Because the powers to be measured are very low, averaging the detected signals over hundreds of laser pulses is absolutely necessary. Then the variation of the backscattered light with the position inside the fiber can be extracted from detection noise. A typical signal measurement averaged over 50,000 shots is shown on figure $2 \mathrm{~b}$ for a 100 ns pulse duration, a peak power of $13 \mathrm{~mW}$ and a $10 \mathrm{~km}$-long fiber. Reflections at both fiber ends are clearly seen, as well as the attenuation along the fiber. An exponential fit from such a measurement results in an evaluation of the attenuation coefficient of $\square \cong 0.05 \mathrm{~km}^{-1} \cong 0.2 \mathrm{~dB} / \mathrm{km}$, which is consistent with values usually determined in telecommunications fibers.

During their 4 hours laboratory work on this set-up, students at École Supérieure d'Optique have to characterize every part of the experiment, from the laser diode to the photodetector. They are thus familiarized with very common components in the field of optical telecommunications. Moreover they are invited to change the laser pulse duration in order to modify the backscattered power and determine experimentally the optimal conditions for the measurements of the attenuation and length of an unknown fiber. Finally, this OTDR set-up highlights the necessity of averaging to detect a signal by improvement of the signal-to-noise ratio as $\sqrt{N}$ (see figures $2 a \& 2 b$ ). The very general problem of the detection of weak and noisy signals is thus emphasized in an easy but significant experiment.

\section{Conclusion}

Our home-made optical time domain reflectometer has very good performances, but remains an educational tool of practical use for our engineer students. During their laboratory work on this set-up, students utilize up-to-date components of the optical telecommunication industry, such as a DFB laser diode or directionnal coupler, that they have to fully characterize. Moreover, this lab work is adapted to show how to detect very low signals, and to sensitize students to the gain/bandwidth compromise of a photodetector. Finally we would like to emphasize the fact that 
this experiment has been realized on two years by three of our students at the master level during a summer traineeship and an experimental school project.

\section{References}

1. Fiber Optic Test and Measurement, Derickson (Prentice Hall, 1998)

2. Optical Fiber Sensors : systems and applications, Culshaw \& Dakin (Artech House, 1989) 